health inequalities and one that is particularly relevant in a worsening macroeconomic climate is that there has been insufficient economic analysis or inadequate adoption of an economic perspective in these areas. The DETERMINE project sought to address this deficit in a discrete work package strand coordinated by the Institute of Public Health in Ireland.

Design Using a specifically designed data collection instrument, examples of economic evaluations conducted on relevant policies and actions were identified and the benefits and barriers to using economic arguments in this area were explored.

Setting Data were collected by partners representing Belgium, Czech Republic, England, Estonia, Finland, Iceland, Netherlands, Northern Ireland, Norway, Poland, Republic of Ireland, Scotland, Slovenia, Spain and Wales. In addition, one partner collected data at the EU level (EuroHealthNet).

Main Outcome Measures The rationale is that where such work has been undertaken, results should be disseminated to influence decision-making in favour of addressing health inequalities. If such work has not been undertaken it is important to understand why this is the case.

Results Findings show that much work is occurring and there are examples of good practice. Barriers and opportunities to progressing work were identified and described by project partners.

Conclusions Economic arguments are a useful approach to tackling health inequalities when used in conjunction with a range of mechanisms. Our research showed diversity across Europe regarding the desire to use such an approach as well as availability of data and tools.

\section{P63 MANDATED ATTENDANCE AT PARENTING WORKSHOPS IMPROVES WOMEN'S HEALTHCARE KNOWLEDGE BUT MAY WIDEN HEALTH INEQUITIES IN LOW AND MIDDLE INCOME COUNTRIES}

doi:10.1136/jech.2010.120477.63

${ }^{1} \mathrm{I}$ Forde, ${ }^{1} \mathrm{~T}$ Chandola, ${ }^{1} \mathrm{M} \mathrm{G}$ Marmot, ${ }^{2} \mathrm{O}$ Attanasio. ${ }^{1}$ Department of Epidemiology and Public Health, University College London, London, UK; ${ }^{2}$ Department of Economics, University College London, London, UK

Background Conditional cash transfer schemes (CCTS) are relatively new policies in low and middle income countries which aim to improve the health and welfare of poor families by investing in their knowledge, skills and resources. Families are offered regular cash as long as they comply with certain conditions. One of these is that mothers/carers attend workshops where parenting and children's healthcare issues are discussed. We hypothesised that presence of a CCTS in Colombia would be associated with an increase in women's healthcare knowledge.

Design and Setting We analysed data from Familias en Acción, Colombia's national CCTS. Households from participating areas and control areas (matched on environmental and socioeconomic criteria) were prospectively surveyed in 2002 and 2006. Women were asked three questions on home-management of acute diarrhoeal illness (ADI), an important cause of childhood mortality for which cost-effective, home-based interventions are available. The sample comprises 5047 women from areas where Familias was operating and 3461 women from control areas.

Outcome Measure and Analysis A marker of healthcare knowledge was constructed from responses to survey items on home-management of ADI, using WHO guidelines to identify correct responses. The effect of Familias was estimated using an ordered probit model, controlling for individual, household and regional characteristics.

Results Familias was associated with nearly three-fold higher workshop attendance $(\times 2.6, \mathrm{p}<0.05)$ and improved knowledge at follow-up (probit coefficient, $\beta=0.160, p=0.010$ ). Multivariate analysis indicated that women's age $(\beta=-0.002, p=0.042)$, literacy $(\beta=0.221, p<0.001)$, level of completed formal education $(\beta=0.260$, $p<0.001)$ and involvement in community activities $(\beta=0.057$, $p=0.033$ ) were independently associated with improved knowledge. Household wealth $(\beta=0.045, p=0.030)$ and head-of-household literacy were also independently associated $(\beta=0.072, \mathrm{p}=0.015)$. Women's literacy $(76 \%)$ did not improve.

Conclusions Familias is associated with increased healthcare knowledge amongst participating women. Women who are younger, literate, better educated, involved in their community and who live in better-off households with a literate head-of-household, however show greater knowledge gain relative to other households. CCTS may widen health inequities by leaving behind women with lowest levels of completed formal education, household resources and community participation. Further thought must be given to the role these workshops play in relation to overall CCTS objectives. The most marginalised households may benefit from additional support to overcome illiteracy and strengthen community involvement.

\section{P64 SOCIAL DETERMINANTS OF DISABILITY AMONG THE IMMIGRANT POPULATION IN CHILE}

doi:10.1136/jech.2010.120477.64

${ }^{1,2} \mathrm{~B}$ Cabieses, ${ }^{1} \mathrm{H}$ Tunstall, ${ }^{1} \mathrm{~K}$ E Pickett. ${ }^{1}$ Department Health Sciences, University of York, York, UK; ${ }^{2}$ Faculdad de Medicine, Universidad del Desarollo, Chile

Introduction Chile is a middle-income country that has experienced progressive economic stability and improvement of its health status, however, one persistent and complex public health problem is disability. Social determinants of health have emerged as a significant aspect of current debates on this health problem. This analysis focuses on the international immigrants, who have not necessarily benefited from the country's economic development.

Objective To compare the social determinants of disability between the international immigrants and the Chilean-born population.

Methods Cross-sectional analysis of a Chilean population-based survey of 268873 people. Independent variable: any disability (visual/hearing/speaking/learning/physical/psychiatric; dichotomous variable). Dependent variables: socio-demographics (age/sex/marital status/geographic location), socio-economics status (3 categories, low/medium/high, by combining income, occupation and education through complete-linkage hierarchical cluster analysis), and material living standards (overcrowding/sanitary conditions/quality of the housing). Descriptive, comparative and adjusted multiple logistic regression models were estimated using STATA 10.0

Results $1 \%$ of the sample reported being an international immigrant and a further $0.67 \%$ did not respond to the question on migration status. International immigrants reported a lower prevalence of disability than the Chilean-born (3.55\% vs $6.93 \%, p<0.001)$; and a clear inverse gradient of disability emerged by immigrants' socioeconomic status. There was a significant association with single status, self-employed/public-sector employees, and being ill and retired $(p<0.001)$. International immigrants living $>20$ years in Chile and those from Peru had a higher chance of disability (OR 2.95 and 7.85, respectively). A protective effect of living in rural areas (OR 0.99) and a negative association with adequate quality of material living standards (OR 0.96) was also found. Those who preferred not to report their migration status showed a higher prevalence of disability than the international immigrants $(7.42 \%, \mathrm{p}<0.001)$, particularly if they were in the poorest income quintile (OR 6.91).

Discussion Social determinants of disability varied between the international immigrants, those who did not respond to the migration status question and the Chilean-born population. Immigrants showed a clear inverse gradient of disability by socioeconomic status. A possible healthy migrant effect was apparent among the international immigrants, but disappeared after 20 years living in Chile. Those who did not respond to the question on migration, potentially undocumented immigrants, showed some 